\title{
Food Act of Sri Lanka: A Systematic Review on Awareness and Practice
}

\section{Charith Amidha Hettiarachchi}

Visiting Fellow, Department of Public Health, Faculty of Heath, University of Technology Sydney, Australia and Post-Doctoral Scholar, Ministry of Health, Sri Lanka

Email: dr.charith@gmail.com; Mobile: +61415587665

\section{ORCHID number: https://orcid.org/0000-0002-7329-6581}

\section{Abstract}

The Food Act of 1980 is the main legislation governing food safety in Sri Lanka. Along with its regulations, the act provides a strong basis to ensure safety of food consumed. However, to achieve its objectives, the act needs to be properly implemented. For that, the food industry needs to adhere to food laws while law enforcing officers need to initiate legal proceedings for law violations. A good knowledge on food laws is needed among food industries, law enforcing officers and consumers contributes towards successive implementation of food laws.

The present review examines the available research literature on knowledge on food regulations and practice of food industries, law enforcing officers (Public Health Inspectors) and consumers in Sri Lanka which helps to identify weaknesses in current law enforcement.

Most of research on the Sri Lankan Food Act of 1980 has focused on food labels. Very few labels have been found to conform with existing laws and some include outrageous health claims. Knowledge and practice of food regulations by food industry, law enforcing officers and consumers in Sri Lanka are not satisfactory which has led to a failure in achieving the intended objectives of the legislature.

\section{Introduction}

The food industry has evolved rapidly in recent decades with the open economy, industrialisation, globalisation, urbanisation and online trade. Food varieties are ever 
increasing, while more and more people rely on commercially available ready to serve food. The dietary pattern is one example of individual behaviour determinants of human health (ODPHP, 2020). The Universal Declaration of Human Rights has identified health as a human right (United Nations, 1948). Further, it has been established that the food consumption pattern is directly related to non-communicable diseases and malnutrition.

Legislation plays an important role in public health. The former Director General of the World Health Organization has stressed the importance of health legislation by stating "there is nothing less than the sovereign right of a nation to enact legislation that protects citizens from harm” (Chan, 2015). The National Nutritional Policy of Sri Lanka has identified making and implementing relevant laws and regulations as a policy to ensure food safety of all Sri Lankans (Ministry of Health, 2010). Therefore, the enforcement of food legislation is important to protect and promote health. Public health, especially in relevant to food safety, cannot be enforced without a strong legal basis.

The first food act in Sri Lanka came in to force in 1949 after gaining the dominion status from British rulers in 1948. It was the Food and Drug Act, No.25 of 1949, which was enacted by the British king under the advice and consent of the Senate and the House of Representatives of Ceylon (Sri Lanka). Sri Lanka gained its full independent in 1972, enabling it to enact laws by Parliament. The Food and Drug Act, No.25 of 1949 was replaced by Food Act, No 26 of 1980. The latter covers only food while predecessor legislation covered both food and drugs. The Food Act of 1980 had been amended twice by Food (amendment) Act, No 20 of 1991 and Food (amendment) Act, No. 29 of 2011. Under section 32 of the Food Act, the minister in charge of health can make regulations in respect to matters required by the Act. To date there are over 40 regulations made including but not limited to the fields of labelling, adverting, registration, sampling, hygiene and standards for specific food.

Availability of food laws itself does not ensure consumers health unless it is properly enforced and monitored. The food industry needs to adhere to food laws while law enforcing officerswho have powers to conduct inspections and prosecutions-need to initiate legal actions for violations. Both food industry and law enforcing officers need to be knowledgeable on food laws in order to implement them. Food laws such as labelling and hygiene regulations are primarily targeted to protect and promote consumer heath; therefore, general knowledge on food laws is beneficial for consumers to ensure consumption of safe food. On the other hand, 
knowledgeable consumers constitute to a strong civil force which help make food laws to be implemented.

Assessment of available research findings on knowledge of food laws among manufactures, law enforcing officers and consumers along with implementation level of food laws by manufactures and law enforcing officers will help to understand the on the ground reality of the outcome of food laws in Sri Lanka.

\section{Objectives}

This review aimed at summarizing the literature on knowledge and practice of food industries, law enforcing officers and consumers in Sri Lanka who are major stakeholders in food safety. It also identifies deficiencies in the food safety legislative system and provides recommendations for further improvements.

\section{Methods}

Post graduate researches in Master's and Doctorate degrees in Community Medicine in Sri Lanka, food legislations in Sri Lanka, national and international journals, websites and online data bases were extensively searched. The research literature was analysed under six headings: Knowledge of Authorized Officers, knowledge of food industry, knowledge of consumers, perceived practice of law enforcing officers, practice of food industry and practice of consumers.

\section{Results}

\section{Knowledge of Authorized Officers}

Knowledge of Authorized Officers on the Sri Lankan Food Act of 1980 and its regulations is essential to commence legal proceedings against offences. In a study conducted among 411 law enforcing officers, none of the Public Health Inspectors (law enforcing officers under the Food Act)) had "very good knowledge” on procedures specific for food legislation while only 6.6\% had "good knowledge" and 48.8\% had "poor knowledge," while the rest had "very poor knowledge.” (Arnold et al., 2005). Inadequate knowledge had been shown in later studies also. In another study, none of the 46 law enforcing officers were competent to identify the main panel of food labels (Hettiarachchi et al., 2017). The same study revealed that more than half could not identify the food label definition of low (52.2\%), medium (52.2\%) and high (58.7\%) sugar levels. However, most of them knew the colour code for low $(84.8 \%$,), medium (80.4\%) and high (78.3\%) sugar levels. Furthermore, 56.5\% of them did not know the diameter of the 
colour circle that is required on labels. Law enforcing officers incorrectly thought that labels of sweetened condensed milks (76.1\%) and drinking yoghurts (34.8\%) should display the colour code for the sugar level even though the Food (Colour Coding for Sugar Level) Regulations, 2016 is not applied to milk-based beverages.

\section{Knowledge of the food industry}

One study assessed the knowledge of manufacturers on labelling regulations. None of the interviewed representatives of manufacturers $(n=134)$ were competent to identify the main panel of food labels. Among 134 manufacturers, more than half did not have correct knowledge on the range of sugar level for lows sugar (53.7\%), medium sugar (57.5\%) and high sugar (53\%) while 53.7\% did not know the correct diameter of the colour circle. However, most of them had correct knowledge on the colour code for low sugar (81.3\%), medium sugar (82.1\%) and high sugar (78.4\%) levels. Furthermore, $75.4 \%$ and $18.7 \%$ of manufacturers had incorrectly thought that labels of sweetened condensed milks and drinking yoghurts should display the colour code for the sugar level respectively (Hettiarachchi et al., 2017). Therefore, the knowledge pattern of manufacturers was similar to law enforcing officers.

\section{Knowledge of consumers}

Food laws are mainly targeted to protect the health of consumers. Few studies have assessed the knowledge of Sri Lankan consumers on labelling laws. Among 542 school children, 88.5\% had good knowledge on the 'best before date'. The rating "good knowledge” was more common on permitted preservatives (88.5\%) than for permitted colourings (52.9\%). However, only few (9.8\%) were able to interpret 'natural product' correctly (Thalagala et al., 2011). In a qualitative study on attitudes towards food labels, two focus group discussions ( $n=10$ and n=9) found that even though consumers mentioned various components of food labels, there appeared to be little awareness that those components are related to labelling regulations (Hettiarachchi et al., 2017).

\section{Perceived practice of law enforcing officers}

It is the responsibility of law enforcing officers (Public Health Inspectors) to initiate legal proceedings under the Sri Lankan Food Act of 1980. A substantial proportion (33.4\%) of Public Health Inspectors admitted that they are not competent regarding court procedures in general and $10.2 \%$ admitted that they cannot perform court procedures (Arnold et al., 2005). A survey conducted in seven provinces in Sri Lanka revealed that a majority of consumers (55.1\%) and law enforcing officers (52.9\%) were not satisfied with the level of enforcement of food safety 
regulations at divisional level. Most consumers thought that law enforcing officers do not perform their duties satisfactorily. A slightly higher proportion of food handlers (55.4\%) stated that the level of enforcement of regulation is satisfactory. A majority of consumers (63\%) and food handlers (42.9\%) stated that there is a potential to improve the level of enforcement (Herath et al., 2004). A later study found that none of the law enforcing officers in Kalutara District had filed a single court case on labelling regulations during the preceding 3 months (Hettiarachchi et al., 2017).

\section{Practice of food industry}

Studies have assessed how well manufacturers have adhered to the Sri Lankan Food Act of 1980 and its regulations. A descriptive cross-sectional study was done in 18 supermarkets in the city of Kandy to assess the implementation of labelling regulations, out of 214 food beverages, $5.1 \%$ did not have the common name in both Sri Lankan languages. Claims or pictures which indicate that the beverage is recommended by medical practitioners or professional associations was present in 8.1\%; 8.9\% claimed that dietary facts benefits heart patients. Out of 64 milk products and green tea, 14\% claimed to be an aid for slimming or weight reduction. All 23 beverages claiming to be enriched did not contain added nutrients (Arnold et al., 2015). All above findings are non-compliance to the provisions of the labelling regulations which indicates poor implementation of labelling regulations by the beverage industry.

Labels ( $\mathrm{n}=835$ ) were also assessed for compliance with labelling regulations in Sri Lanka. None of the labels were 100\% compliant with of Food (Labelling and Advertising) Regulations of 2005, Food (Colour Coding for Sugar Level) Regulations of 2016, Food (Standards) Regulations of 1989, Food (Bottled and Packed Water) Regulations of 2005, Food (Sweeteners) Regulation of 2003, and Food (Flavouring Substances and Flavour Enhances) Regulations of 2013. In other words, all food labels were illegal (Hettiarachchi et al., 2017). Even though not fully compliant with all provisions, among 292 carbonated and fruit-based beverages, $88.7 \%$ displayed the numerical description of the sugar content, the relative sugar level, and the colour code while the same proportion displayed numerical descriptions. However, in $61.8 \%$ of labels the font size of the relative sugar level was less than $1.5 \mathrm{~mm}$ in at least one language. Further, the diameter of the colour circle was less than 10mm in 21 labels (8.1\%) (Hettiarachchi et al., 2017). 
Among 152 dried milk products, the mandatory statement “(type of dried milk) milk, this tin contains the equivalent of (quantity) pints of milk" was present in 59.6\%, while the statement "to make a fluid not below the composition of (type of dried milk) add (quantity) by weight of this powder to (quantity) of water” was on only 32.9\%. Among the 141 products where these statements were available, only $24.1 \%$ adhered to the correct font type and background colour; $25.2 \%$ of these statements were surrounded by a line-as required by the law (Hettiarachchi et al., 2017). Further, among 89 bottled or packed water products, two labels (2.2\%) misled the consumer on the nature, origin, composition, and properties by a statement or picture. The words "permitted sweeteners" were not included in $21.9 \%$ of labels where sweeteners were listed under ingredients. Of 4 labels with aspartame on the ingredient list, 3 did not have the warning "not recommended for children.” The words "natural (name of the flavour) flavour" and "artificial (name of the flavour) flavour" were not correctly stated in $43.8 \%$ and $16.7 \%$ labels respectively when flavours were indicated in the ingredient list; however, the wording "nature identical (name of the flavour) flavour" was correctly used on all labels (Hettiarachchi et al., 2017). Clearly, manufactures are not properly following the food laws in Sri Lanka.

\section{Practice of consumers}

Some studies have assessed how consumers have applied knowledge of various labelling components at the time of purchase. A community based cross sectional study was carried out among grade 12 students in four government schools in the Colombo district to assess the use of food labels on making choices of packaged foods/drinks. Oof the 542 students, $75 \%$ did not use the label completely. However, when consuming a packaged snack for the first time, more than $70 \%$ paid attention 'always' or 'most of the time' to the expiry date, manufactured date, price, brand name and nutrition panel (Thalagala et al., 2011).

The impact of labelling on consumer buying behaviour in Kandy was assessed in a crosssectional study which selected 90 consumers randomly from three supermarkets. Consumers' awareness on nutritional labelling, the effect of nutrition labelling on buying decisions and willingness to pay for nutrition label information were assessed. 65\% bought products only after reading nutrition information, mainly due to health consciousness (Prathiraja et al., 2003). Another descriptive cross sectional study was conducted on labelling of genetically modified foods in Sri Lanka at two supermarkets in Kandy and Colombo. Out of 120 participants, 87\% read labels regularly while 12\% read "sometimes" and 1\% "did not read at all” (Senerath et al., 2010). 


\section{Problems with legislation}

Having a multitude of regulations falling under the Sri Lankan Food Act of 1980 on the same subject area is likely to confuse food industry and law enforcing officers. The Legislature, under the guidance of the Health Ministry, has failed to amalgamate similar regulations enacted over long periods of time. Provisions relevant to one specific component are scattered throughout regulations rather than organized under subheadings.

There are no dedicated Sri Lankan Public Health Inspectors; rather they are also allocated for communicable diseases, non-communicable diseases, school health and environmental health programmes. Thus, implementation of the Food Act is only one part among their many other functions. In addition, there are no standard training packages available in Sri Lanka on food legislation for either the food industry or law enforcing officers.

Another possible reason for poor practice among Sri Lankan law enforcing officers is the failure of proper supervision by the Food Authorities. The Food Authority has the prime responsibility to ensure the enforcement of the Food Act and the regulations in its administrative area. The Municipal Council is the Food Authority in the Municipal Council area. As the minister has not appointed any other local authority, the Medical Officer of Health is the Food Authority for urban and rural local government areas in Sri Lanka (Government Publication Bureau, 1980). Since a majority of the country is under the urban and rural council areas, the Medical Officers of Health are the Food Authorities in a majority of areas in the country. Medical Officers of Health who are the immediate supervising Food Authority, are not specifically designated for food safety activities. They also provide their services in other health areas like maternal and child health, immunization, communicable and noncommunicable diseases and occupational/environmental health. Most of the time of Medical Officers of Health are busy conducting maternal clinics in the field. Therefore, it is not surprising that food legislation activities are poorly supervised by the Food Authorities.

\section{Discussion}

Only a few studies have been done relevant to the Sri Lankan Food Act of 1980, and among them most related to food labelling legislations. This indicates that the food legislation is one of study areas in Sri Lanka which have been least assessed by scientific research.

Reasons for the non-compliance to food legislation is a two-way fault: one is with the food industry for failing to comply and the other is with the law enforcing officers for failing to learn about the laws and execute them. As per the Sri Lankan Food Act of 1980, every person who 
contravenes any of the provisions of the Act or regulations made thereunder or fails to comply with any direction given by law enforcing officers shall be guilty of an offence, while it is the duty of law enforcing officers to prosecute for offences (Government Publication Bureau, 1980). In addition, the Manual of Public Health Inspectors in its Public Health Legislation Chapter describes the Public Health Inspector as a person who handles the legal proceedings of public health legislation, carrying out responsibilities of law enforcing officers under the Food Act (Ministry of Health, 2010). Sri Lanka is a country with a near 100\% literature rate. Hence, the strict implementation of food legislation will invariably improve the health of people which in turn may reduce the non-communicable disease burden. Studies have suggested that implementation of food legislation is poor in Sri Lanka compared to other legislative and administrative regulations.

A single regulatory document for each subject area with organized subheadings would be beneficial to food industry as well as to law enforcing officers in Sri Lanka. Food Safety and Standards (Packaging and labelling) Regulations of 2011 which is the Indian labelling regulation (FISSAI, 2020) is such an example where all labelling requirements stipulated under a single document with well-organized subheadings. Codex Food Standards too has arranged provisions under subheadings, making them user friendly and easy to understand (Codex, 2015). All these factors have led Sri Lankan regulations to their inherent complexity. This may have led to non-adherence food legislation by food industry as well as lesser number of prosecutions by law enforcing officers. Many countries have identified the importance of food regulations and some like Australia and India have specifically designated officers to implement food laws. Some have also developed programmes to improve knowledge through both state and non-state organizations. For example, online learning courses on food legislation have been developed in United Kingdom (Food Standard Agency, 2020).

\section{Conclusions and Recommendations}

Only limited research has been conducted relevant to the Food Act of 1980 of Sri Lanka. Available research suggests that the implementation or enforcement of the Sri Lankan Food Act of 1980 by both food industries and law enforcing officers is not satisfactory. In addition, both food manufacturers and law enforcing officers do not have satisfactory knowledge on food regulations. Reasons for poor implementation of food legislation are multifactorial with inadequate training, unavailability of specifically designated officers to enforce the law, and inadequate monitoring may have been contributed. The author wishes to recommend standard user-friendly short training courses on food legislation, improvement of supervision of law 
enforcing officers and allocation of specifically designated officers to food safety and law enforcement activities. Awareness of school children and the general public on basic food laws specially related to food hygiene and labelling also needs to be increased.

\section{Competing interests}

No conflicts of interests

\section{Funding}

No funding was necessary.

\section{Acknowledgements}

Authors wish to acknowledge Post Graduate Institute of Medicine, University of Colombo, Sri Lanka, Ministry of Health Sri Lanka, University of Technology Sydney, Australia.

\section{References}

Arnold, S. M. 2005. An intervention study to improve the implementation of specific legislation related to food safety by Public Health inspectors. MD Thesis, Post Graduate Institute of Medicine, University of Colombo.

Arnold, S. M., Wickramatilake, M. S. K., Fernando, R. M. S. D., Denawake, C. J., Fernando, W. Y. J., Aluthge, K. C. P. Suraweera, P. J. 2015. Compliance of health and nutrition related claims of beverages to the Sri Lankan Food Labelling Regulation. Annual Academic Sessions of College of Community Physicians of Sri Lanka. Colombo, Sri Lanka.

Chan, M. (2015). Director General of the World Health Organization, lecture delivered at George Town Universities Global Futures Initiative, Washington, USA. https://www.who.int/dg/speeches/2015

Codex Alimentarius Commission, 2015. Food Standards.

https://www.who.codexalimentarius.net.com

Food Safety and Standard Authority of India, 2020. Food Safety and Standards (Packaging and Labelling) Regulation, 2011. https://www.fssai.gov.in/cms

Food Standard Agency, 2020. Business guidance. https://www.food.gov.uk/businessguidance

Government Publication Bureau, 1980. Food Act of 1980. Sri Lanka: Government Publication Bureau.

Herath, H. D. B. 2004. Food safety activities at divisional level in selected provinces. Post Graduate Institute of Medicine, University of Colombo. 
Hettiarachchi, C. A, Arnold, S. M, Nandasena, M. 2017. Level of adherence of labels of carbonated beverages to Sri Lankan Food Regulations 2016. Journal of the College of Community Physicians of Sri Lanka, 23(3):47-48.

Ministry of Health, 2010. National Nutritional Policy. Colombo: Ministry of Health

Ministry of Health, 2010. Manual for the Sri Lanka Public Health Inspector. Colombo: Ministry of Health

ODPHP, 2020. Determinants of Health. https://www.healthypeople.gov

Prathiraja, P. H. K, Ariyawardana, A. 2003. Impact of Nutritional Labeling on Consumer Buying Behavior. Sri Lankan Journal of Agricultural Economics, 5:35-46.

Thalagala, I.A. 2011. The use of food labels in making choices on package snacks and its associated factors among grade 12 students in the district of Colombo. MSc dissertation, Post Graduate Institute of Medicine, University of Colombo.

United Nations, 1948. Universal Declaration of human rights. https://www.un.org.com 